\title{
Primary invasive lobular carcinoma arising in mammary-like glands of the vulva managed with neoadjuvant trastuzumab- based chemotherapy, excision, and sentinel lymph node biopsy
}

\author{
Shiva Niakan $^{1}$ (D) | Heather Love ${ }^{1}$ | Qing Cao ${ }^{2}$ | Nada Kawar ${ }^{1}$
}

\author{
${ }^{1}$ Department of Obstetrics and Gynecology, \\ University of Massachusetts Medical \\ School-Baystate, Springfield, MA, USA \\ ${ }^{2}$ Department of Pathology, University of \\ Massachusetts Medical School-Baystate, \\ Springfield, MA, USA \\ Correspondence \\ Shiva Niakan, Department of Obstetrics and \\ Gynecology, University of Massachusetts \\ Medical School-Baystate, 759 Chestnut St, \\ Springfield MA 01199. \\ Email: Shiva.NiakanDo@baystatehealth.org
}

\begin{abstract}
A rare case of Her2/neu-positive mammary-like gland adenocarcinoma of the vulva treated with neoadjuvant therapy, trastuzumab, and achieved complete pathological response on excision, adjuvant radiation, complicated by brain metastasis recurrence.
\end{abstract}

\section{K E Y W O R D S}

obstetrics and gynaecology, oncology

\section{1 | INTRODUCTION}

Mammary-like glands (MLG) are normal structures in the vulva which have possible but rare malignant potential. ${ }^{1}$ Given this rarity, guidelines for treating MLG adenocarcinoma are lacking. In the current literature, approximately thirty-six reported cases of MLG in the vulva exist.

There are currently no guidelines for the management of primary vulvar mammary-like gland carcinoma given its rare incidence. The primary source of literature on this topic is found in case reports and from extrapolation from guidelines for treatment of normally located breast cancer and include surgery, chemotherapy, endocrine therapy, and radiation. This case describes a 55-year-old woman with a vulvar mass diagnosed as Her2/neu-positive primary infiltrating lobular breast carcinoma. She subsequently underwent neoadjuvant treatment with Her2/neu directed therapy, achieving a complete pathological response upon excision, followed by adjuvant radiation with course complicated by brain metastasis recurrence.

\section{2 | CASE PRESENTATION}

A fifty-five-year-old gravida 1, para 0010 woman presented with nontender, firm mass on the left labia noted to have enlarged over the course of three months. Examination notable for 4-centimeter, firm, discoid mass of left labium majus with small overlying skin ulceration, with no inguinal lymphadenopathy (Figure 1A). Remainder of the physical examination including that of breasts was unremarkable. Punch biopsy of vulvar lesion revealed infiltrating carcinoma (Figure 2A), with mostly noncohesive tumor cells in single lines or thin cords, with high nuclear grade. Tumor cells stained immunohistochemically positive for GATA3 (Figure 2B), indicating breast tissue differentiation. The tumor cells were negative for estrogen receptor (ER) and progesterone receptor (PR), but strongly positive for Her2/neu (Figure 2C). It was best classified as invasive lobular carcinoma, pleomorphic type.

Patient's breast cancer workup including bilateral mammography, breast ultrasound, and MRI were unremarkable with no evidence of primary malignancy in either breast. 
F I G URE 1 A: Pelvic examination on initial presentation; B: Pelvic examination postneoadjuvant therapy
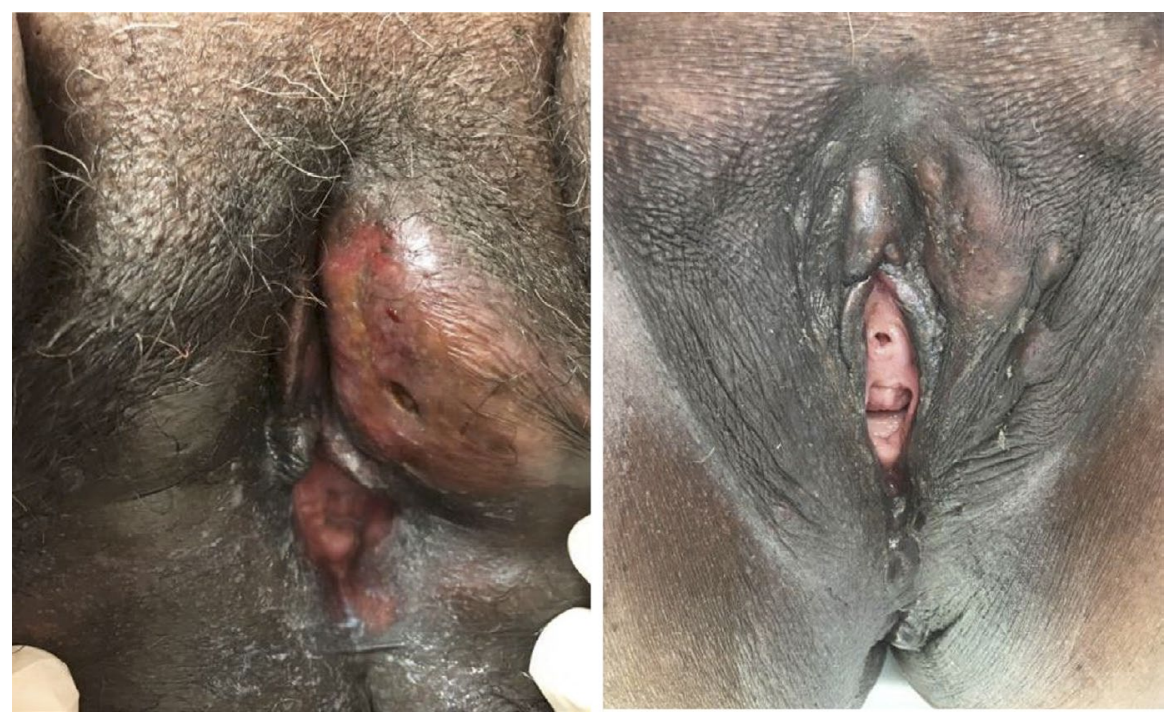

FIG URE 2 H\&E stain of invasive lobular carcinoma arising from mammarylike glands in the vulva, pleomorphic type (A). Immunohistochemical stain with GATA3 (B), Her2/neu (C) in invasive carcinoma of vulva, and GATA3 in metastatic carcinoma in the brain (D)
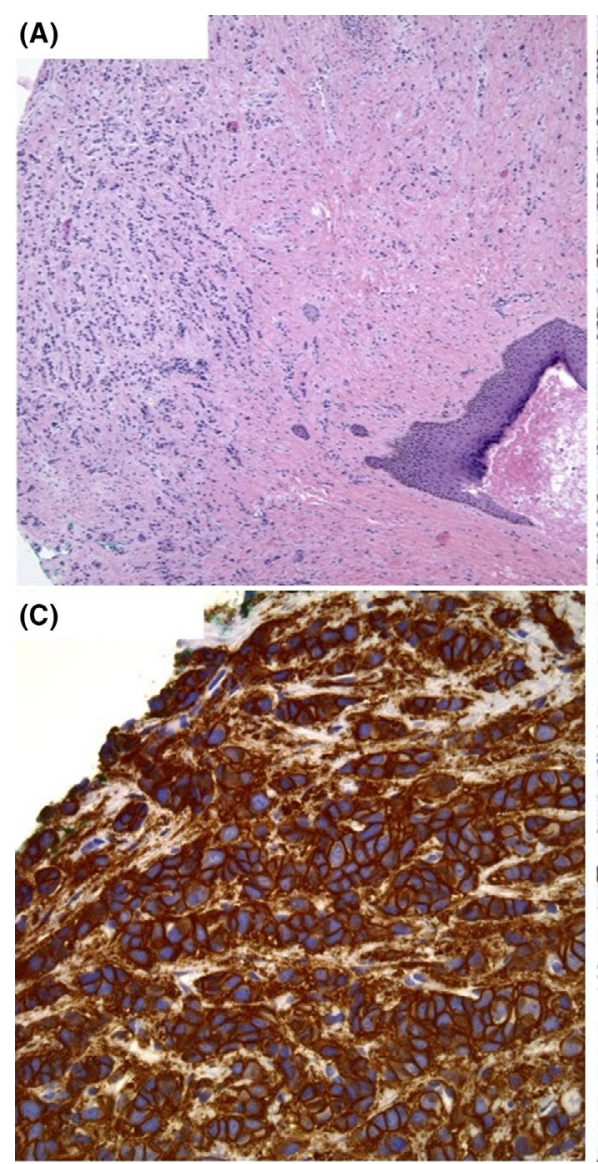

Given these findings, her tumor was best considered as primary invasive breast cancer arising in mammary-like glands of the vulva.

Patient received neoadjuvant chemotherapy consisting of carboplatin, docetaxel, trastuzumab, and pertuzumab with a good clinical response (Figure 1B). This was followed by left partial radical vulvectomy with left inguinal sentinel lymph node biopsy. Pathology revealed a $3.5-\mathrm{cm}$ tumor bed with dense fibrosis and scattered inflammatory cells with no residual carcinoma identified. Within the tumor bed, benign mammary-like glands immunohistochemically positive for GATA3 and estrogen receptors were also present. Three sentinel lymph nodes were negative for metastasis by H\&E stains and antikeratin immunohistochemical study. Therapy with trastuzumab given Her2/neu status was continued, and the patient completed fifteen of seventeen planned cycles.

Two years following initiation of chemotherapy, the patient presented to the emergency department with weakness, 
change in gait, and dizziness. Brain CT and MRI demonstrated cerebellar mass and compression with resulting hydrocephalus. She underwent craniotomy with resection of mass, and pathology revealed metastatic carcinoma with similar morphology and immunohistochemical profile (positive for GATA3 and Her2/neu) consistent with her prior vulvar infiltrating lobular carcinoma (Figure 2D). Her postoperative course consisted of physical rehabilitation, and stereotactic radiosurgery (SRS). To date, four years following her initial diagnosis, she has completed her course of radiation and is now undergoing Her2/neu directed therapy with lapatinib and capecitabine. Recent imaging demonstrates no new lesions or metastatic progression.

\section{3 | DISCUSSION}

Mammals are characterized by the unique presence of mammary glands, with two mammary lines extending from the axilla to the inguinal region. In humans, however, normal development of mammary glands is limited to pectoral regions. ${ }^{2}$ It had long been believed that if portions of the mammary ridges do not regress, these remnant cells can become ectopic breast tissue later in life, which may have malignant potential. $^{3}$

In 1872, Hartung described the first case of "ectopic breast tissue" found in the vulva; since then, many more cases have been reported. In 1991, van der Putte coined the term "mammary-like glands of the vulva" to describe what used to be called anogenital "sweat glands." Mammary-like glands (MLG) are a mixture of eccrine and apocrine glands almost identical to breast parenchyma. He also postulated that the development of breast tissue in humans is actually not similar to other mammals, and that according to studies of human embryos, the milk lines do not extend beyond the axillary-pectoral line. Furthermore, in embryological development, the human breast and vulva are so vastly separated both by distance and developmental time that MLG cannot be remnant cells derived from "milk lines." It is fascinating that MLG has eccrine origin, yet shares many characteristics with breast tissue including similar histopathological features and immunohistochemical markers with estrogen, progesterone, and Her2/neu. This explains why vulvar lesions of MLG are also seen in the breast fibroadenoma, extramammary Paget's disease, hidradenoma papilliferum, lactating adenoma, and adenocarcinoma. $^{5}$

Diagnosis of carcinoma arising from MLG of the vulva is based on histopathological features, with status of estrogen, progesterone, and Her2/neu receptors. This is not enough, however, to distinguish primary versus metastatic disease. Breast cancer workup with mammogram and MRI can help to make the distinction. In the case presented here, the patient had no lesion at breast proper. The presence of GATA3positive MLG in the vulvar and brain tumor bed confirmed mammary-like glands origin.

Approximately $15-19 \%$ of all breast cancers overexpress Her $2 /$ neu. $^{6}$ The amplification of this protein is significant as it is associated with more aggressive disease, higher recurrence rate, and overall lower survival. ${ }^{7}$ Treatment with trastuzumab, a monoclonal antibody that targets Her2/neu, drastically improves the prognosis of Her2/neu-positive breast cancer and has been shown to decrease risk of recurrence by $50 \%{ }^{6}$ The primary chemotherapy utilized in this case has been trastuzumab. This is the first case report utilizing therapy directed at Her2/neu-positive primary vulvar breast cancer.

Review of the literature revealed 36 cases of mammary-like carcinoma or ectopic breast tissue arising in the vulva (Table 1). The average age was 61.8. Tumors were often tested for estrogen, progesterone, and Her2/neu status. The incidence of Her2/neu in reported cases of MLG is consistent with the incidence reported in breast cancer. Treatments were similar to primary breast cancer with combinations of endocrine therapy with tamoxifen and aromatase inhibitors, chemotherapy, surgery, including sentinel lymph node biopsy, and radiation therapy (Table 2 ).

\section{4 | CONCLUSION}

Although very rare, MLG carcinoma of the vulva should be included in the differential for vulvar tumors. Biopsy of the lesion followed by pathology, receptor testing, and immunohistochemical testing are essential in making the diagnosis and initiating the appropriate treatment course. It is also crucial to complete a workup for metastatic breast cancer with diagnostic imaging.

Recent literature indicates that mammary-like glands are in fact normal vulvar structures with anogenital eccrine gland origin. Although mammary-like glands are not believed to be ectopic breast tissue, they remain similar to breast tissue, and thus, current data suggest treating MLG carcinoma similarly to primary breast cancer. This case illustrates the multidisciplinary management utilizing an approach similar to primary breast carcinoma, including neoadjuvant chemotherapy with trastuzumab and pertuzumab, sentinel lymph node analysis, and treatment of brain metastatic disease with radiotherapy, and systemic treatment with lapatinib and capecitabine.

\section{ACKNOWLEDGMENTS}

Dr. Seth Kaufman and Dr. Deborah Katz for proofreading, and language editing. Published with written consent of the patient. 
T A B L E 1 Summary of reported cases of mammary-like glands of the vulva

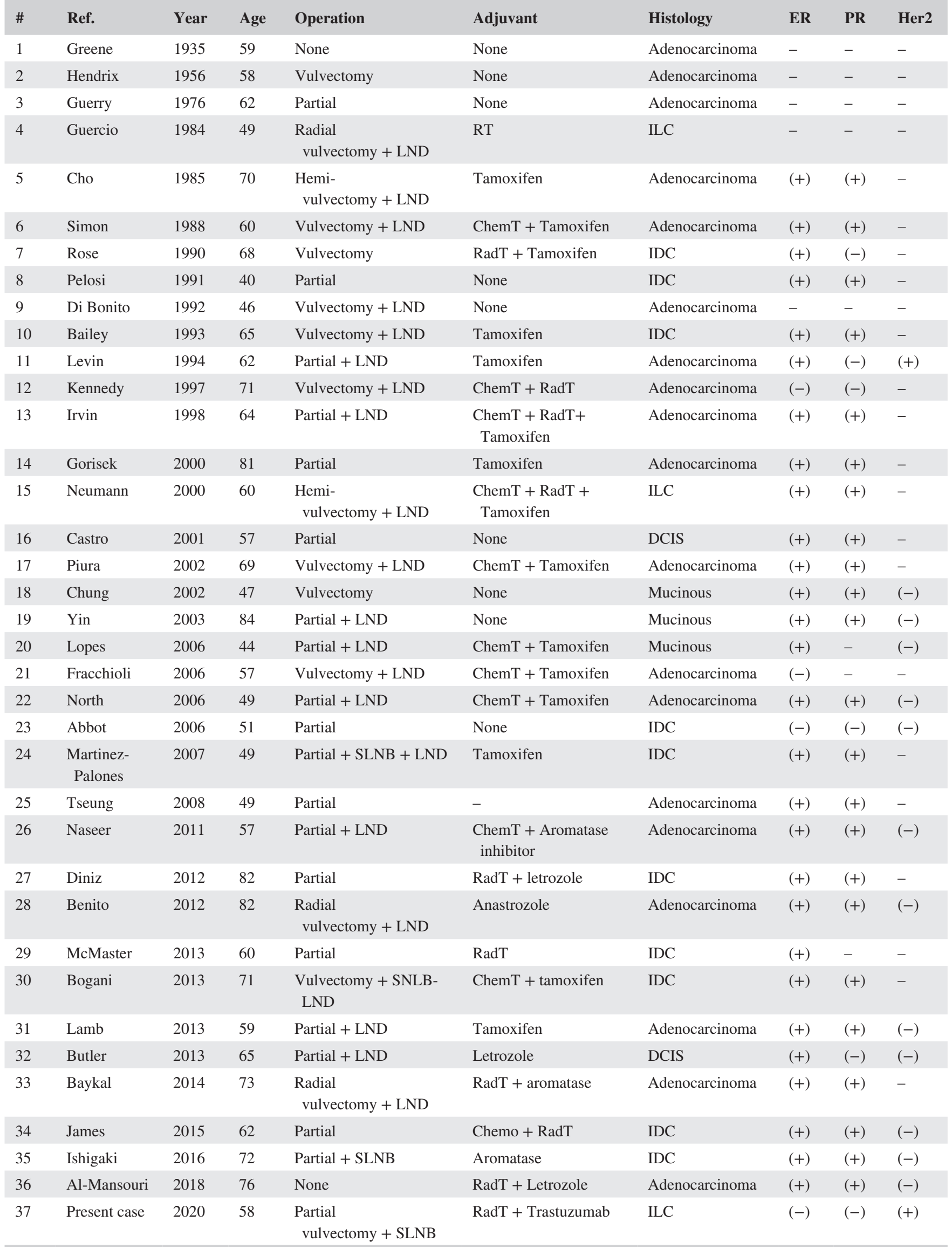


T A B LE 2 Characteristics of mammary-like glands of the vulva

\begin{tabular}{|lll|}
\hline Receptor & Ratios $^{\mathbf{a}}$ & Percentage $^{\mathbf{a}}$ \\
\hline $\mathrm{ER}+$ & $28 / 32$ & $87 \%$ \\
\hline $\mathrm{PR}+$ & $23 / 29$ & $79 \%$ \\
\hline $\mathrm{ER}+/ \mathrm{PR}+$ & $23 / 29$ & $79 \%$ \\
\hline Her2/neu + & $2 / 13$ & $15 \%$ \\
\hline Triple negative & $1 / 13$ & $8 \%$ \\
\hline Adjuvant therapy & & \\
\hline Tamoxifen & $15 / 37$ & $42 \%$ \\
Chemotherapy & $11 / 37$ & $31 \%$ \\
Radiation & $11 / 37$ & $31 \%$ \\
None & $10 / 37$ & $28 \%$ \\
Aromatase Inhibitor & $7 / 37$ & $17 \%$ \\
Combination & $16 / 37$ & $44 \%$ \\
\hline Histopathological Types of MLG Carcinoma & \\
IDC & $29 / 37$ & $78.4 \%$ \\
DCIS & $2 / 37$ & $5.4 \%$ \\
ILC & $3 / 37$ & $8.1 \%$ \\
Mucinuous & $3 / 37$ & $8.1 \%$ \\
\hline
\end{tabular}

MLG, mammary-like gland; IDC, invasive/infiltrating ductal carcinoma, also includes adenocarcinoma; DCIS, ductal carcinoma in situ; ILC, invasive/ infiltrating lobular carcinoma.

${ }^{\mathrm{a}}$ Indicates ratios and percentage for those patients for which these receptors were tested.

\section{CONFLICT OF INTEREST}

None declared.

\section{AUTHOR CONTRIBUTIONS}

SN: served as first author, drafted manuscript, revised for intellectual content, made substantial contribution to design, completed literature review, and interpretation, administrative support, prepared images and tables, and accountable for work submitted. HL: drafted manuscript, revised for intellectual content, made substantial contribution to design, proofreading, interpretation of data, and accountable for work submitted. JC: made substantial contribution to conception of manuscript, design, proofreading, analysis and interpretation of data, and revision of manuscript critically for intellectual content, gave approval for final version to be published, and accountable for work submitted. NK: made substantial contribution to conception of manuscript, design, proofreading, analysis and interpretation of data, revision of manuscript critically for intellectual content, gave approval for final version to be published, and accountable for work submitted.

\section{DATA AVAILABILITY STATEMENT}

Data sharing not applicable—no new data generated.

\section{ORCID}

Shiva Niakan (D) https://orcid.org/0000-0002-2402-8943

\section{REFERENCES}

1. Abbott J, Ahmed I. Adenocarcinoma of Mammary-Like Glands of the Vulva. Am J Dermatopathol. 2006;28(2):127-133.

2. North J, Perez D, Fentiman G, et al. Primary Breast Cancer of the Vulva: Case Report and Literature Review. Aust N Z J Obstet Gynaecol. 2007;47(1):77-79.

3. Marshall M, Moynihan JJ, Frost A, Evans SR. Ectopic Breast Cancer: Case Report and Literature Review. J Surg Oncol. 1994;3(5):295-304.

4. van der Putte S, van Gorp L. Adenocarcinoma of the mammary-like glands of the vulva: a concept unifying sweat gland carcinoma of the vulva, carcinoma of the supernumerary mammary glands and extramammary Paget's disease. J Cutan Pathol. 1994;21:157-63.

5. Kazakov D, Spagnolo D, Kacerovska D, Michal M. Lesions of anogenital mammary-like glands: an update. Adv Anat Pathol. 2011;18(1):1-28.

6. Cronin K, Harlan LC, Dodd KW, Abrams JS, Ballard-Barbash R. Population-based estimate of the prevalence of HER-2 positive breast cancer tumors for early stage patients in the US. Cancer Invest. 2010;28(9):963-968.

7. Ross J, Slodkowska EA, Symmans WF, Pusztai L, Ravdin PM, Hortobagyi GN. The HER-2 Receptor and Breast Cancer: Ten Years of Targeted Anti-HER-2 Therapy and Personalized Medicine. Oncologist. 2009;14(4):320-368.

How to cite this article: Niakan S, Love H, Cao Q, Kawar N. Primary invasive lobular carcinoma arising in mammary-like glands of the vulva managed with neoadjuvant trastuzumab-based chemotherapy, excision, and sentinel lymph node biopsy. Clin Case Rep. 2021;9:118-122. https://doi.org/10.1002/ccr3.3475 